\title{
Store-and-Forward Performance in a DTN
}

\author{
Mooi-Choo Chuah, Peng Yang, Brian D. Davison, Liang Cheng \\ Department of Computer Science and Engineering \\ Lehigh University \\ 19 Memorial Drive West, Bethlehem, PA 18015, USA \\ \{chuah, pey204, davison, cheng $@$ cse.lehigh.edu
}

\begin{abstract}
Delay and disruption tolerant networks have been proposed to address data communication challenges in network scenarios where an instantaneous end-to-end path between a source and destination may not exist, and the links between nodes may be opportunistic, predictably connectable, or periodically(dis)connected. In this paper, we describe the store-and-forward and custody transfer concepts that are used in DTNs. Then, we present simulation results that illustrate the usefulness of the custody transfer feature, and a message ferry in improving the end-to-end message delivery ratio in a multihop scenario where link availability can be as low as $\mathbf{2 0 \%}$. In particular, our results indicate that one can achieve a delivery ratio as high as $\mathbf{9 0 - 9 9 \%}$ with appropriate buffer allocations. We also provide some preliminary insights on the design factors that influence the end to end delivery ratio, e.g., the link availability patterns and buffer allocation strategies.
\end{abstract}

Keywords-disruption tolerant networks; custody transfer; route discovery, message ferry

\section{INTRODUCTION}

Packet-switched network communication has been studied for decades. Important progress has been made in robustness and scalability in the TCP/IP protocol suite based primarily on principles of end-to-end protocols and services [6]. However, there are many scenarios in which an end-to-end connection is not guaranteed or even possible, and so an intermediary is needed, perhaps to translate between protocols or to provide temporary storage (e.g., in mail servers). In these cases, without such intermediaries, communication would fail. In other cases, communication may fail not because of a lack of instantaneous connection, but because the connection properties fall beyond the expected bounds (excessive roundtrip-time or high packet loss probability).

Solutions have been proposed to deal with some specific situations, e.g., using link layer retransmissions to deal with high packet loss probability in wireless environments [1]. However, these solutions still do not work in situations where there are no end-to-end paths. Recently, a considerable amount of research focusing on delay/disruption-tolerant networking and communications has been published (e.g., [10],[12]). Diesel [11] is a disruption tolerant network where connections between nodes are short-lived and occasional. A common

This work is sponsored by Defense Advanced Research Projects Agency (DARPA). Any opinions, findings, and conclusions or recommendations expressed in this material are those of the authors and do not necessarily reflect the views of DARPA. This document is approved for public release, unlimited distribution. approach used to address delays and disruptions is via the use of a store-and-forward mechanism similar to electronic mail [8]. This makes communication possible, even when an instantaneous end-to-end path does not exist

In [2], Fall describes an architecture for delay tolerant networking that implements much of what we have described. It proposed the idea of topological regions connected by gateways, which were responsible for storing messages in nonvolatile storage to provide for reliable delivery. End-point addressing in his scenario consisted of a region name used for inter-region routing and a locally-resolvable name for intraregion delivery. More recently, we have proposed an enhanced disruption-tolerant network architecture called EDIFY (Enhanced Disruption and Fault Tolerant Bundle Delivery) [6]. Our approach builds on many ideas from Fall, but adds support for multiple, overlapping name spaces and node and group mobility.

In this paper, however, we are concerned with how the store-and-forward approach (fundamental to any DTN architecture) performs in an environment with link availability as low as $20 \%$, with and without the custody transfer feature and the presence of a message ferry. Using ns-2 simulations, we find that the combination of the custody transfer feature, the use of a message ferry, and sufficient per-node storage allows a high message delivery ratio (around $90-99 \%$ ) to be achieved between groups of nodes that are intermittently connected.

In what follows, we first give an overview of our enhanced DTN architecture in Section 2. We describe the different network entities present in our architecture. We then describe the route discovery process, and the custody transfer feature in EDIFY. In Section 3, we describe the simulation experiments that were conducted to demonstrate the impacts of link availability patterns on multihop intergroup communications in DTN environments. We also provide simulation results to demonstrate the extent of performance improvements one gets using a message ferry. We conclude with some future work in Section IV.

\section{THE EDIFY DTN ARCHITECTURE}

In the EDIFY architecture, there are several types of nodes, namely, (a) regular DTN nodes, (b) the DTN name registrar (DNR), and (c) DTN gateway. Regular DTN nodes have the ability to send to, and receive bundles from other nearby nodes. Each DTN node may be configured with a default DNR from which each node acquires its name within the group. Every node in the DTN has one or more names - the DTN address 
within a group to which it belongs. An address comprises of two parts - a hierarchically-organized group name shared with all other members, and a group-specific name that is unique among all members.

In some DTN networks, some nodes take on additional roles and responsibilities. An example is the DNR. A DNR node functions as the administrative node for a group and is responsible for communications with the parent group(s). A DNR offers the mandatory service of registering the members and visitors. It is responsible for ensuring the authenticity and eligibility of the nodes requesting to be registered. DNRs from different groups may form an overlay network. DTN gateways are DTN nodes that offer forwarding services to one or more destination groups. DTN nodes that perform forwarding services for different groups form the communication backbone within the DTN. A DTN gateway can advertise its services to the local registrar so that nodes in the group can identify which DTN gateway to use by asking the registrar or the DTN gateway can flood its local group with forwarding service advertisements. A message ferry is a special type of DTN gateway that moves around to allow communications between different groups that are not within transmission range of one another. Regular DTN nodes may form an ad hoc DTN network without the presence of a DNR. In an ad hoc DTN network, the DTN nodes behave similarly to the regular wireless ad hoc networks except that the DTN nodes support DTN functionalities like custody transfer which will be described in a later subsection.

\section{A. Route Discovery in EDIFY}

Via an example with a message ferry, we describe how the nodes in a DTN environment can discover routes to other nodes. In Fig. 1, we have forty nodes that are partitioned into four isolated groups. There is a base station node in each group. The base station node is assumed to have a second long range radio that provides a wireless backhaul link with higher bandwidth. Due to potential enemy detection, the wireless backhaul links are only turned on periodically for short durations of time. In the example shown in Figure 3, we assume that BS1 (BS2) can communicate only occasionally with BS2 (BS3). Similarly, BS3 can communicate only occasionally with BS4.

Because these groups are isolated far away from one another, the groups can only communicate with one another either via the wireless backhaul links that are not always available or via a message ferry. We assume that the message ferry broadcasts a service announcement message periodically as it moves along a fixed route. We also assume the service announcement message contains information on the groups that the message ferry can reach from previous trips. Other useful information like the estimated next visit time to those reachable groups may be included for more sophisticated forwarding decision-making.

We assume that the intragroup routing protocol makes use of the information provided by the underlying ad hoc routing protocol which is assumed to be DSR-like [4]. Whenever there is intergroup traffic, the nodes will evaluate to see if it consumes less cost (e.g., in terms of expected delivery delay) to send the traffic via the backhaul links or via the message ferry if both types of forwarding services are available. The base station will send announcements to inform the nodes whether or not it can provide intergroup forwarding services. For example, when Link 1 is not available, BS1 will inform all group members 1 that intergroup service is not available. Similarly, when Link 2 is not available, BS2 will inform BS1 that it cannot communicate with Group 3. Then, BS1 will inform Group 1 members that intergroup service to Group 3 is not available. Note that BS2 can delay such notification until its buffers are full or can notify BS1 immediately when Link 2 disappears.

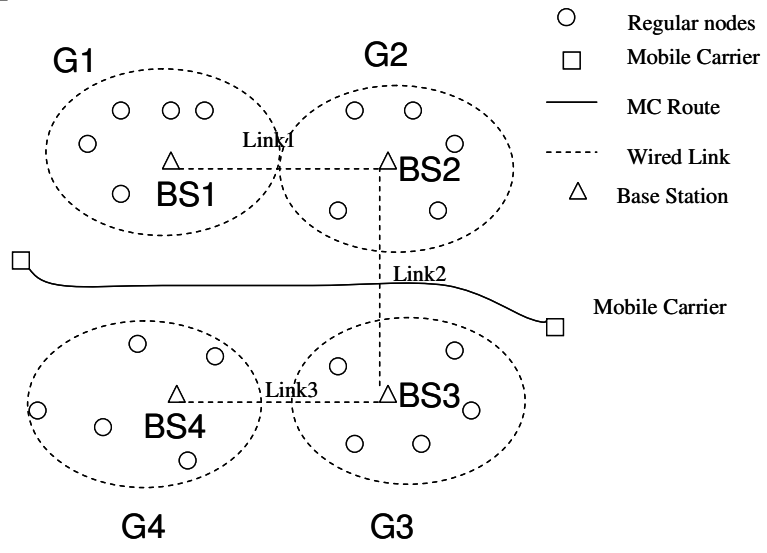

Figure 1. A 4-group DTN example

Assume that Group 1 needs to communicate with Group 3; a source node (which will be a node in Group 1) will send the traffic to BS1 and BS1 will forward it to BS2 when link 1 is available. If Link 2 is not available, the messages will be stored at the buffers at BS2 until Link 2 is available. Dropfrom-front scheme can be used to replace old messages with new messages when the buffer at a base station is full. However, one can also use tail-drop scheme where no new messages will be accepted when the buffer at the base station is full. In addition, when different classes of messages are available, smarter buffer management schemes need to be designed to give different treatments to messages from different classes.

When the base station does not provide intergroup service or if the cost for sending such traffic using the backhaul route is higher than using a route via the message ferry, the regular nodes will use the service from the message ferry. Not all nodes can hear the message ferry. We assume that all nodes within a group that can hear the service announcement from a message ferry can provide forwarding services to/from the message ferry. Such nodes will be referred to as gateway nodes. Gateway nodes can make periodic announcements to their group members that they can provide forwarding services. All regular nodes can cache information from such announcements but do not use the gateway nodes until they desire to use the service of the message ferry. The above approach is more proactive since the nodes within a group can find out where the gateway nodes are before they need to use the forwarding services from such nodes. Alternatively, the sending nodes can send gateway discovery messages to discover the gateway nodes. Once a sending node can identify 
the gateway node, it can then send intergroup traffic to the gateway node. This approach is more reactive and message delivery time may increase due to the need to perform gateway discovery.

\section{B. Custody Transfer}

A custody transfer feature is proposed in [10],[14] to provide communications in a intermittently connected network. In their proposal, accepting a message with custody transfer amounts to promising not to delete it until it can be reliably delivered to another node providing custody transfer or it arrives at the destination. Nodes holding a message with custody are called custodians. Normally, a message has a single custodian (referred to as sole custody) but in some circumstances, more than one custodian owns a message or message fragment (referred to as joint custody). Applications can optionally request the custody transfer feature on a permessage basis and they will receive a custody acknowledgement when their host system can find one or more nodes that are willing to take custody of the message. A node may agree to accept custody for messages initially and refuse to do so when its local node resources, e.g., buffers, become substantially consumed.

\section{Simulation STUDiES}

Using the network topology shown in Figure 3, we conduct extensive simulation experiments to evaluate the impacts of the custody transfer feature, and the use of a message ferry on the message delivery ratio when the availabilities of the wireless backhaul links are varied both in terms of the relative on-off patterns and the percentages of their availabilities. We also explore the impacts of having limited buffers at the base stations and regular DTN nodes on the end-to-end message delivery ratio. We use ns-2 [5] for our simulations. The common parameter values used in the simulation are tabulated in Table 1. Each group has ten nodes which are randomly distributed over an area of $1000 \mathrm{~m}$ by $1000 \mathrm{~m}$. Thus, each group forms a sparse ad hoc network. All nodes support DTN functionalities. We assume that the regular DTN nodes communicate with one another via the 802.11 links with 2 Mbps link bandwidth, while the wireless backhaul link has a bandwidth of $5 \mathrm{Mbps}$. We assume that only one type of message is used and that the message has a fixed size of 512 bytes. A complete message is sent using one bundle. We further assume that the message ferry has a buffer size of 400 messages. For each experiment, we measure the delivery ratio for the messages delivered via the wireless backhaul links and the message ferry separately. We also measure the contact time a message ferry has with a particular group during its route to help us understand the delivery ratio in each experiment. In addition, we also record the end-to-end message delivery times.

The implemented custody transfer feature works as follows: when a DTN node has a message which it owns custodianship to send, it checks its cache to see if it has a route to the destination node. If it finds more than one route, it picks the one with the lowest cost (e.g., using hop count, delivery latency etc., as metrics). When a route is selected, it checks the DTN nodes included in this selected route to see which node is the best candidate for custody transfer, e.g., the closest DTN node that has buffer space available. Then, it sends a custodian request to that downstream DTN node. If the DTN node can accept the custodianship, it will respond with a custody acknowledgement. Otherwise, it sends a negative reply.

If the sending DTN node cannot find a route to the destination of the message, it will trigger its underlying ad hoc network layer to look for a route or neighboring nodes that are closer to the destination than itself. In addition, it will send a custody request message at the DTN layer. The DTN nodes that hear the custody request message will send a custodian accept message to the sender of that request if it has available buffers. At the ad hoc network routing layer, all DTN nodes that receive a route reply message with the DTN option flag set will set a bit in the appropriate position (according to its hop distance from the sending node of the route request) to indicate buffer availability before relaying the route reply message. That way, the sender knows whether or not that it can use that route. In a DTN environment, an end-to-end route may not exist. Thus, our dual-layer (at ad hoc network routing and DTN layers) approach allows us to identify downstream nodes to which we can forward the messages.

There are 10 pairs of traffic sessions where 4 pairs are single hop pairs (meaning requiring a traversal of only one backhaul link for delivery), 4 pairs are 2-hop pairs (meaning requiring a traversal of two backhaul links for delivery), and 2 pairs that are 3-hop pairs (meaning it needs to traverse 3 backhaul links) when the message ferry service is not available.

TABLe 1: Common Simulation PaRAmeter VALUeS

\begin{tabular}{|l|l|}
\hline Parameter & Value \\
\hline Simulation Areas & $2000 \mathrm{~m}$ x 2000m \\
\hline Group Size & 10 nodes/group \\
\hline Wireless Link & 802.11 (2Mbps) \\
\hline Wired Link & duplex link (5Mbps) \\
\hline Packet Size & 512bytes/packet \\
\hline Traffic Pattern & CBR (interval:4sec/packet) \\
\hline Buffer Size of Regular Nodes & Depending on experiments \\
\hline Buffer Replacement Policy & Drop-from-front \\
\hline Buffer of the Mobile Carrier & 400 messages \\
\hline Speed of the Mobile Carrier & $15 \mathrm{~m} / \mathrm{s}$ \\
\hline Traffic Load & 10 pairs \\
\hline Simulation Time & 5000 seconds \\
\hline
\end{tabular}

\section{A. Impact of custody transfer on delivery ratio}

In our first set of experiments, we investigate how the custody transfer feature helps in the message delivery ratio. The backhaul link follows an on/off pattern shown in Fig 2 (Case 1) with a mean on/off cycle time of 100 seconds. We set the base station and the message ferry buffer size to be 400 messages. The buffer size for regular DTN nodes is set to 100 or 200 messages. We simulated four scenarios, namely, a) backhaul delivery without custody transfer, (b) backhaul delivery with custody transfer, (c) ferry delivery without custody transfer, and (d) ferry delivery with custody transfer. The availability of the backhaul links follows the Case 1 on/off 
pattern illustrated in Figure 4. The message delivery ratios achieved in these four scenarios are tabulated in Table 2. Our results show that the custody transfer feature improves the message delivery ratio significantly to $90-92 \%$ with only the backhaul delivery mechanism and $89.4 \%$ (with 200 message buffers) with only the ferry delivery mechanism. The lower message delivery ratio for the message ferry case is due to the highly disruptive intragroup routes to the gateway nodes since each group is a sparse ad hoc network.

TABLE 2: Message DeliVERY RATIO FOR EXPERIMENT 1

\begin{tabular}{|l|l|l|}
\hline Buffer Size & 100 & 200 \\
\hline Backhaul-delivery without custody-transfer & $55.6 \%$ & $58.4 \%$ \\
\hline Backhaul-delivery with custody-transfer & $90.9 \%$ & $92.3 \%$ \\
\hline Ferry-delivery without custody-transfer & $10.3 \%$ & $11.6 \%$ \\
\hline Ferry-delivery with custody-transfer & $78.6 \%$ & $89.4 \%$ \\
\hline
\end{tabular}

\section{B. Impact of Link Pattern on End-to-end Message Delivery}

In this experiment, we fix the link availability to be $20 \%$ but vary the link availability patterns of the 3 links to investigate its impacts on the message delivery ratio. The 3 link patterns we use are shown in Figure 4. The on/off times follow an exponential distribution with a certain mean on/off times to mimic link patterns shown. In Case 1 and Case 2, each link is available for an average of 20 seconds and not available for an average of 80 seconds. The two cases only differ in the relative positions of the link availabilities. To achieve the on/off patterns shown as Case 1 in Figure 6, we generate a random on time for link 1 (say x1), then schedule for link 2 to be on only after time $\mathrm{t} 1$ and it will be on for another random on time (say $\mathrm{x} 2$ ), etc. In Case 3, each link is available for an average of 30 sec and not available for an average of $120 \mathrm{sec}$. So, the link availability is also $20 \%$.

In our second experiment, we set the mean on/off period to be 200 seconds. The custody transfer feature is turned on. The DTN nodes only use the backhaul links (no message ferry service is provided). The results are tabulated in Table 3 . The number expressed in seconds is the mean end-to-end message delivery time. There are a few interesting observations we can make from these results. First, the delivery ratio for the traffic session between Group 1 and Group 3 (indicated by 1-3 pair) is lower than other pairs because the route between the source node and the base station breaks more frequently than other traffic pairs, e.g., Group 3 to Group 1. The mean end-to-end delivery time depends on various factors, e.g., the link availability pattern, the connectivity between the source node and the base station, etc. For example, even though session 1-3 and session 3-1 are both 2-hop pairs, the average end-to-end message delivery time for session 3-1 is higher than that achieved for session 1-3 using Case 1 link patterns because the traffic from Group 3 requires at least two on/off cycles to reach Group 1 but the traffic from Group 1 to Group 3 only needs one on/off cycle. In general, the mean end-to-end message delivery times are higher for traffic sessions that traverse more backhaul hops. However, there may be situations where this is not true. For example, the mean end-to-end delivery time for traffic session 1-4 is smaller than the mean end-to-end delivery time for traffic session 1-3. This is due to the fact that the source node for this 1-3 pair happens to be sparsely connected to the base station in Group 1 while the source node for the 1-4 pair has a very reliable route to the base station in Group 1.
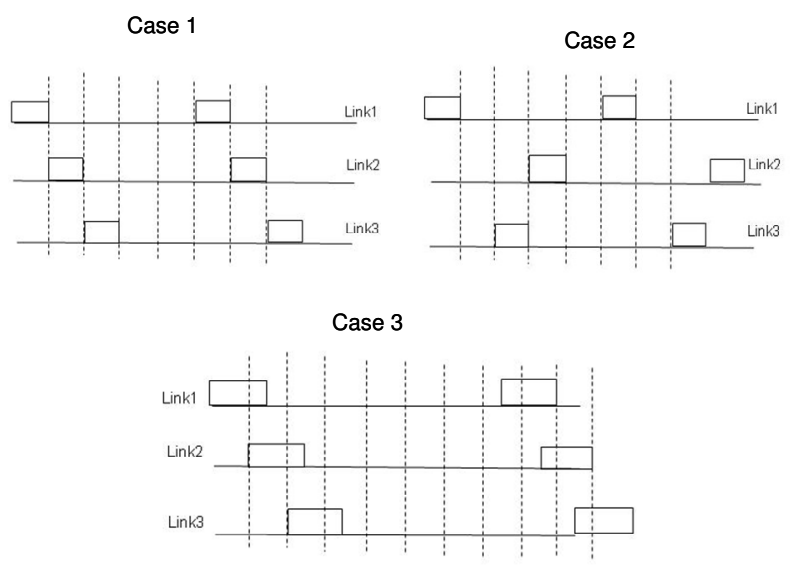

Figure 2. Various Link Availability Patterns

Table 3: Message Delivery Ratio and MeAn End-TO-End Message DELIVERY TIME FOR EXPERIMENT 2.

\begin{tabular}{|c|c|c|c|}
\hline & Link Pattern 1 & Link Pattern 2 & Link Pattern 3 \\
\hline 1-3 pair & $78.7 \%$ & $82.0 \%$ & $75.1 \%$ \\
& 277.2 seconds & 410.8 seconds & 385.9 seconds \\
\hline 3-1 pair & $86.7 \%$ & $84.7 \%$ & $82.1 \%$ \\
& 352.5 seconds & 337.1 seconds & 306.4 seconds \\
\hline 2-4 pair & $96.2 \%$ & $97.0 \%$ & $99.1 \%$ \\
& 131.4 seconds & 278.3 seconds & 144.5 seconds \\
\hline 4-2 pair & $96.0 \%$ & $97.0 \%$ & $96.9 \%$ \\
& 229.3 seconds & 145.9 seconds & 166.9 seconds \\
\hline 1-4 pair & $75.9 \%$ & $77.4 \%$ & $79.3 \%$ \\
& 188.1 seconds & 432.0 seconds & 239.3 seconds \\
\hline 4-1 pair & $85.0 \%$ & $96.8 \%$ & $72.2 \%$ \\
& 444.6 seconds & 240.9 seconds & 345.7 seconds \\
\hline 1-hop & $88.7 \%$ & $89.4 \%$ & $87.9 \%$ \\
pairs & 138.5 seconds & 148.3 seconds & 193.7 seconds \\
\hline
\end{tabular}

\section{Buffer Size Study}

In the third experiment, the nodes use either (a) only the backhaul links, (b) only the message ferry, or (c) both backhaul links and the message ferry to deliver intergroup traffic. We set the message ferry and base station buffer size to be 400 messages each. We then vary the buffer size of the regular DTN nodes to see what its impact on the message delivery ratio and end-to-end message delivery times. A mean on/off cycle of 200 seconds is used for this third experiment. The results for the message delivery ratio, the end-to-end message delivery time (denoted as delay in Figure 4) and the overhead of control messages sent are plotted in Figures 3, 4, and 5. The results indicate that a delivery ratio of $90 \%$ is achievable even with $20 \%$ link availability.

\section{CONCLUSIONS}

New network scenarios are challenging the assumptions of Internet service models. In such scenarios, an instantaneous end-to-end path between a source and destination may not exist, and the links between nodes may be opportunistic, 
predictably connectable, or periodically (dis)connected. We have proposed an enhanced disruption tolerant network (EDIFY) architecture to address such challenges [3].

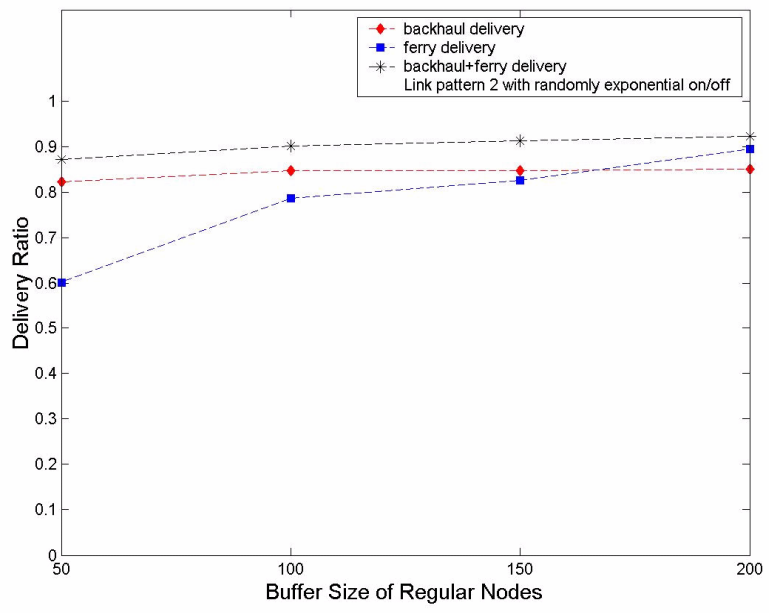

Figure 3: Message Delivery Ratio

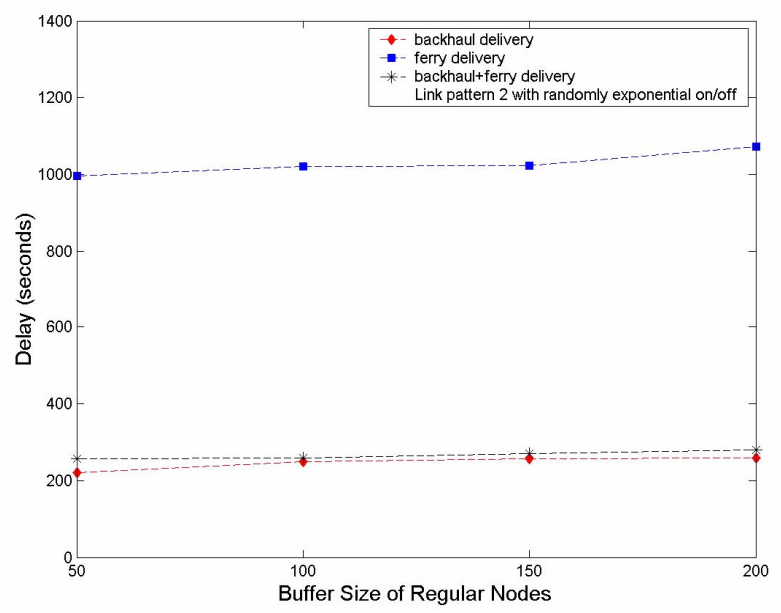

Figure 4: End-to-end Message Delivery Time

In this paper, we illustrate the usefulness of the custody transfer feature and a message ferry in improving the end-toend message delivery ratio in a multihop scenario where link availability can be as low as $20 \%$. In particular, our results indicate that one can achieve a delivery ratio as high as $90-99 \%$ with appropriate buffer allocations. We also provide some preliminary insights on the design factors that influence the end to end delivery ratio, e.g., the link availability patterns and buffer allocation strategies. We do not explore the issue of joint custodianship in this paper. This is left for future work. In addition, we assume in this paper that the traffic demands from one group to another do not vary with time and that the link availability follows exponential on/off distribution. In real world scenarios, the traffic demands and the link availabilities may be changing dynamically so one may not be able to predict the maximum required buffer size for the base station. So, more intelligent distributed buffer management schemes still need to be designed to achieve high delivery ratio for DTN environments. We leave such topics for future research.

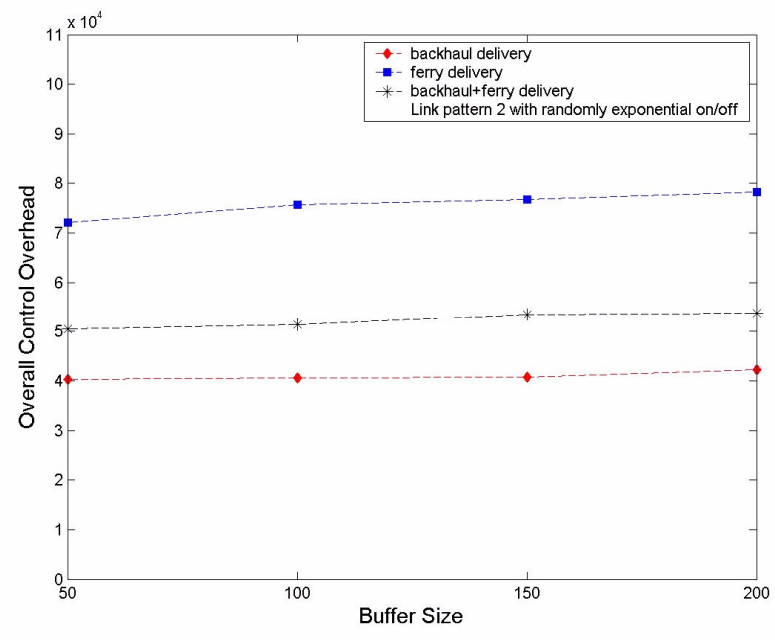

Figure 5: Control Overhead

\section{REFERENCES}

[1] H. Balakrishnan, V. N. Padmanabhan, S. Seshan, and R. H. Katz, "A comparison of mechanisms for improving TCP performance over wireless links", IEEE/ACM Transactions on Networking, 5(6):756-769, December 1997.

[2] K. Fall, "A delay-tolerant network architecture for challenged Internets", Proceedings of ACM SIGCOMM, August 2003.

[3] M. Chuah, L. Cheng, and B. D. Davison, "Enhanced Disruption and Fault Tolerant Network Architecture for Bundle Delivery (EDIFY)", Proceedings of IEEE Globecom, November 2005

[4] C. E. Perkins and E. M. Royer, "Ad-hoc On-Demand Distance Vector Routing", Proceedings of IEEE Workshop on Mobile Computing Systems and Applications, pp 90-100, Feb 1999.

[5] UCB/LBNL/VINT, "The Network Simulator ns-2", Online at http://www.isi.edu/nsnam/ns/

[6] J. H. Saltzer, D. P. Reed, and D. D. Clark, "End-to-End Arguments in System Design", ACM Transactions on Computer Systems, 2(4):277-288, November 1984

[7] J. Klensin, Editor, "Simple mail transfer protocol", IETF RFC 2821, April 2001.

[8] R. Viswanathan, J. Li, and M. C. Chuah, "Message Ferrying for Constrainted Scenarios", poster, Proc. of WoWMoM, 2005.

[9] W. Zhao and M. H. Ammar, "Message Ferrying: Proactive Routing in Highly Partitioned Wireless Adhoc Networks", Proceedings of the IEEE Workshop on Future Trends in Distributed Computing Systems, Puerto Rico, May, 2003.

[10] V. Cerf et al., "Delay-Tolerant Network Architecture", Internet Draft, draft-irtf-dtnrg-arch-04.txt, December 2005.

[11] B. Burns, O. Brock and B. N. Levine, "MV Routing and Capacity Building in Disruption Tolerant Networks", Proceedings of IEEE Infocom, March 2005.

[12] W. Zhao, M. Ammar, and E. Zegura, "A message ferrying approach for data delivery in sparse mobile ad hoc networks", Proceedings of ACM Mobihoc, May 2004.

[13] W. Ma and M. Chuah, "Comparisons of Interdomain Routing Schemes for Heterogeneous Adhoc Networks", Proceedings of WoWMoM, 2005.

[14] K. Fall, W. Hong and S. Madden, "Custody transfer for reliable delivery in delay tolerant networks", IRB-TR-03-030, July 2003. 